\title{
Circulating tumor DNA: new horizons for improving cancer treatment
}

\author{
“The concept of circulating tumor DNA-based next-generation \\ sequencing analyses will revolutionize the management of cancer \\ patients with potentially curable or metastatic disease."
}

\section{Georgios D Lianos ${ }^{1,2}$, Alberto Mangano ${ }^{3}$, William C Cho ${ }^{4}$, Gianlorenzo Dionigi ${ }^{3}$ \& Dimitrios H Roukos ${ }^{*, 1,2,5}$}

Recent cancer progress has been too slow $[1,2]$. Resistance to current therapeutics represents the major problem [3]. More recent developed technique of circulating tumor DNA (ctDNA) [4] detection and subsequently next-generation sequencing (NGS) application in these 'fluid' biopsies provides a partial explanation of therapeutic resistance due to the emergence of mutation. This mutational emerging heterogeneity assessment shapes a new roadmap for more effective therapeutic development [3].

Recent evidence suggests that progress in management of patients with advanced and metastatic cancer remains modest over the last decade. Although the use of novel sophisticated technologies is a reality, little progress has occurred in clinical oncology. Recent data on tumor and patient 'diversity' shape now an exciting pathway in achieving the desired goal of a true personalized medicine for cancer patients [5]. We have to keep in mind that although current oncological practice is based on standardized single biopsy-based approach, serious important limitations in predicting resistance to current therapies and tumor progression are existing.

\section{Circulating tumor DNA}

A new biobanking sequency strategy on multiple biopsy-based genome represents a novel important tool in cancer genomics. In this editorial article, we address ctDNA clone-based strategy for predicting and treating therapeutic resistance $[4,6-8]$. The concept of ctDNA-based NGS analyses will revolutionize the management of cancer patients with potentially curable or metastatic disease. During and after treatment, repeated ctDNA detection and NGS analyses could potentially predict recurrence before it clinically occurs. Thereby, targeting the emergency mutations with inhibitors of specific oncogenic pathways we would potentially prevent recurrence [4]. Similarly, in the metastatic setting, we could administrate agents which can more effectively treat the emergency mutated genes $[5,6]$.

Moreover, latest evidence highlights that the future of personalized cancer medicine is represented by interpatient and intratumor 'diversity.' Different genetic landscape and variations among patients with the same tumor, clinical and pathological properties and staging

\section{KEYWORDS}

- cancer $\bullet$ circulating tumor DNA

- clonal evolution • genetic variation

- next-generation sequencing analyses $\bullet$ personalized medicine

- recurrence $\bullet$ regulatory networks

- tumor heterogeneity

“...progress in management of

patients with advanced and metastatic cancer remains modest over the last decade." 
“...we can achieve important genetic information of diverse types of tumors even in an early stage with a simple blood sample." are intended by interpatient heterogeneity. Moreover, different mutational background in 'subpopulations' of cancer cells in different geographical places of the same tumor is defined intratumor heterogeneity (ITH) $[3,6,9]$. Today, the investigators, by exploring the mechanisms underlying the wide ITH status with the important help of new noninvasive tools such as ctDNA, may revolutionize the future of medicine and may significantly improve the survival rates of cancer patients with the discovery of novel drugs that will overcome resistance to therapy.

\section{Decision-making oncological therapy}

Decision-making therapy by oncologists is usually based on a single biopsy obtained from primary tumor after surgical resection or single needle biopsy. Latest data support that a single biopsy-based DNA sequencing test is not enough to obtain all the necessary genetic information [10]. Recent genome sequencybased data suggest that the whole genetic background may represent a useful tool for predicting the response or resistance to targeted therapy. There is no doubt that cancer is a 'complex' process and unfortunately the heterogeneity-diversity of the genetic background among patients and tumors cannot be adequately evaluated by a single surgical biopsy [11]. Interpatient heterogeneity and ITH nowadays represent a real medical challenge, because if they can be 'characterized' and assessed, they may lead to better primary systemic treatment. Notably, the most critical issue in terms of decision making during tumor progression is represented by intratumor 'diversity' of the primary tumor subclonal cell populations [10-12]. In the same patient, the metastatic progress of the tumor or the resistance to therapy can be better managed when its heterogeneity is assessed. However, multiple tumor biopsies from the metastatic sites of the disease in the majority of cases are very invasive and difficult to be performed. We have also to keep in mind that in the absence of metastasis there is no accurate predictive method of resistance to therapy and recurrence. But now, we can achieve important genetic information of diverse types of tumors even in an early stage with a simple blood sample. In the near future these exciting methods may lead to predict resistance to therapy or recurrence even in early-stage cancer [12-15].

\section{Proof-of-concept on circulating tumor DNA}

It is a common topic now that multiple repeated tumor biopsies are often nonfeasible and more invasive. In this way, Murtaza et. al [4] in a recent study performed whole-exome sequencing of plasma DNA in six patients with advanced cancers. There were two cases with breast cancer, three cases with ovarian cancer and one case with non-small-cell lung cancer. Whole-exome sequencing was performed on multiple samples of plasma from each patient. It is reported that the followup was up to 665 days (range: 109665 days, median: 433 days). The researchers used $4-20 \%$ of the DNA extracted from 2.0$2.2 \mathrm{ml}$ of plasma, and an average of $169 \mathrm{mil}-$ lion reads of sequencing per sample. They have analyzed the coding exons of all proteincoding genes at an average unique coverage depth ranging from 31- to 160 -fold. For two cases, synchronous biopsies were also analyzed, confirming genome-wide representation of the tumor genome in plasma. To identify changes in the mutation profiles of the tumors, they have compared the abundance of somatic mutations found in plasma before and after each course of systemic therapy. In this way, they have shown that exome analysis of plasma ctDNA represents a novel noninvasive tool for characterization of tumor progress. The researchers involved in this innovative study strongly support that ctDNA are representative of the tumor genome and provide a valid alternative novel method of tumor sampling without the need of multiple invasive tumor biopsies [4,14,15]. What is more, new genes or important mutated genetic pathways maybe identified with this novel method.

Few months ago, Bettegowda et al. [16] published a very important and innovative study. Their aim was to compare many different types of cancer and to determine in which cancers ctDNA detection levels could represent a useful clinical tool. The researchers in this study have measured and evaluated an important number of tumor types. In this way, they have purified plasma and tumor DNA using specific protocols for all the tumor samples and they have used advanced technological methods to detect and evaluate ctDNA levels from each tumor. Notably, they were able to report the number of mutant templates per milliliter of plasma in every tumor type. They have enrolled in this exciting study 136 metastatic tumors from 14 different tissues, as well as 41 patients with 
primary brain tumors such as glioma and medulloblastoma. Additionally, they have included ten cases of stage III ovarian and hepatocellular carcinomas. The investigators showed that most patients with stage III ovarian and liver cancers and metastatic cancers of the pancreas, bladder, colon, stomach, breast, liver, esophagus, head and neck, as well as patients with neuroblastoma and melanoma, harbored detectable levels of ctDNA. On the other hand, less than 50\% of patients with medulloblastomas, metastatic kidney, prostate or thyroid cancers and less than $10 \%$ of patients with gliomas, harbored detectable ctDNA levels. Moreover, we have to keep in mind that although ctDNA was detectable in most patients with metastatic cancers, the ctDNA's concentration varied among patients. Moreover, ctDNA's concentration varied also among patients with the same tumor type.

The most important aspect of this study was the evaluation of ctDNA levels in patients with early-localized disease. The researchers specified that with the term of localized disease it is intended a 'disease status' where there is no clinical or radiological sign of distant metastasis at the time of sample collection. We have to highlight the fact that among 223 patients with localized cancers of all types, the researchers showed that detectable levels of ctDNA were found in 55\% of the patients (122 of 223 patients). Differences in the rate of patients with detectable levels of ctDNA were also correlated with the stage. In this way, $47 \%$ of patients with stage I cancers of any type had detectable ctDNA, whereas the fraction of patients with detectable ctDNA was 55, 69 and $82 \%$ for patients with stage II, III and IV cancers, respectively. Bettegowda et al. [16] concluded after this innovative study of 640 patients, that mutant DNA fragments are found at lower but detectable levels in patients with early-stage localized cancers and in high levels

\section{References}

1 Vogelstein B, Papadopoulos N, Velculescu VE et al. Cancer genome landscapes. Science 339(6127), 1546-1558 (2013).

2 Klein CA. Selection and adaptation during metastatic cancer progression. Nature 501(7467), 365-372 (2013).

3 Bedard PL, Hansen AR, Ratain MJ, Siu LL. Tumour heterogeneity in the clinic. Nature 501(7467), 355-364 (2013).

4 Murtaza M, Dawson SJ, Tsui DW et al. Non-invasive analysis of acquired resistance to in the circulation of patients with advancedstage cancers. We will expect that this research team or other scientific groups, in addition to ctDNA detection will also perform NGS analysis in this large number of samples. It is out of question that these amazing results may have several scientific implications and may suggest novel important pathways of future translational research [15].

Taken all these data together, novel noninvasive biomarkers may provide many advantages as compared with invasive multiple tumor biopsies. The above-mentioned approach of ctDNA represents a novel exciting strategy for reaching personalized modern medicine [4,16-17]. Therefore, NGS-based analysis of ctDNA could be used as a valid biomarker for cancer monitoring. Recent improvements in NGS methods and ctDNA method could lead to great expectations in the translation of research into clinical personalized cancer medicine [5,17]. This ctDNA-based genomic diversity identified by NGS analyses also opens a new way for more accurate and effective therapeutic strategy with a combination of targeted drugs against the complete set of genome changes [18-21]. However, it should be emphasized that new clinical trials will be required to prove the robustness of these biomarkers and effectiveness of this therapeutic strategy.

\section{Financial \& competing interests disclosure}

The authors have no relevant affliations or financial involvement with any organization or entity with a financial interest in or financial conflict with the subject matter or materials discussed in the manuscript. This includes employment, consultancies, honoraria, stock ownership or options, expert testimony, grants or patents received or pending, or royalties.

No writing assistance was utilized in the production of this manuscript. cancer therapy by sequencing of plasma DNA. Nature 497(7447), 108-112 (2013).

5 Jurgensmeier JM, Eder JP, Herbst RS. New strategies in personalized medicine for solid tumors: molecular markers and clinical trial designs. Clin. Cancer Res. 20(17), 4425-4435 (2014).

6 Gerlinger M, Rowan NJ, Horswell S et al. Intratumor heterogeneity and branched evolution revealed by multiregion sequencing. N. Engl. J. Med. 366(10), 883-892 (2012).
Pantel K, Diaz LA Jr, Polyak K. Tracking tumor resistance using 'liquid biopsies'. Nat. Med. 19(6), 676-677 (2013).

8 Chan KC, Jiang P, Zheng YW et al. Cancer genome scanning in plasma: detection of tumorassociated copy number aberrations, single-nucleotide variants, and tumoral heterogeneity by massively parallel sequencing. Clin. Chem. 59, 211-224 (2013).

9 Leary RJ, Sausen M, Kinde I et al. Detection of chromosomal alterations in the circulation of cancer patients with whole-genome sequencing. Sci. Transl. Med. 4, 162 (2012). 
EDITORIAL Lianos, Mangano, Cho, Dionigi \& Roukos

10 Leary RJ, Sausen M, Diaz LA Jr, Velculescu VE. Cancer detection using whole-genome sequencing of cell free DNA. Oncotarget 4(8), 1119-1120 (2013).

11 Neph S, Stergachis AB, Reynolds A, Sandstrom $\mathrm{R}$, Borenstein E, Stamatoyannopoulos JA. Circuitry and dynamics of human transcription factor regulatory networks. Cell 150(6), 1274-1286 (2012).

12 Tran B, Dancey JE, Kamel-Reid S et al. Cancer genomics: technology, discovery, and translation. J. Clin. Oncol. 30, 647-660 (2012).

13 Greaves M, Maley CC. Clonal evolution in cancer. Nature 481, 306-313 (2012).
14 Polzer B, Klein CA. Metastasis awakening: the challenges of targeting minimal residual cancer. Nat. Med. 19, 274-275 (2013).

15 Swanton C. Intratumor heterogeneity: evolution through space and time. Cancer Res. 72(19), 4875-4882 (2012).

16 Bettegowda C, Sausen M, Leary R et al. Detection of circulating tumor DNA in early- and late-stage human malignancies. Sci. Transl. Med. 6, $224 r a 24$ (2014).

17 Lawrence MS, Stojanov P, Mermel CH et al. Discovery and saturation analysis of cancer genes across 21 tumour types. Nature $505,495-501$ (2014).
18 Lappalainen T, Sammeth M, Friedländer MR et al. Trascriptome and genome sequencing uncovers functional variation in humans. Nature 501(7468), 506-511 (2013).

19 Hanahan, D, Weinberg RA. Hallmarks of cancer: the next generation. Cell 144(5), 646-674 (2011).

20 Aparicio S, Caldas C. The implications of clonal genome evolution for cancer medicine. N. Engl. J. Med. 368, 842-851 (2013).

21 Rask-Andersen M, Almén MS, Schiöth HB. Trends in the exploitation of novel drug targets. Nat. Rev. Drug. Discov. 10(8), 579-590 (2011). 